\title{
Structured Fuzzy Based Methodological Approach towards Sustainability Performance Assessment
}

\author{
P. O. St Flour, T. Makoondlall-Chadee, C. Bokhoree, and R. Mohee
}

\begin{abstract}
Sustainability has become a fundamental element for most countries in this new era. Its concept is found to be very complex and difficult to measure. As it is essential to obtain relevant information to take long term management decision, sustainability should be measured urgently. The subject is treated using an appropriate technique namely the fuzzy approach. It further seems appropriate to deal with ambiguity and vague situations where mathematical formula is ineffective. The model describes how fuzzy logic can be applied in measuring development sustainability using indicators. The model intends to treat inputs from individual components (economic, environmental and social) and they are combined using fuzzy rule bases to provide an overall measure of sustainability. It may then be addressed to scientists and policy makers as they move towards a sustainable path in the near future.
\end{abstract}

Index Terms-Fuzzy logic, indicators, sustainability performance assessment, sustainability.

\section{INTRODUCTION}

In recent years, sustainability has become an area of interest for policy makers and scientists both at national and international level. Sustainability refers to objectives to be achieved with sustainable development referring to the process to achieve them [1]. Strictly speaking sustainability is the synonym for sustainable development. The understanding of this concept was viewed as a one-dimensional paradigm where researches were undertaken mainly environmentally or socially or economically based but future research has revealed that the three dimensions should be interrelated and be examined more in depth [2]. Concerns about some various detrimental side effects identified include climatic changes, soil erosion, poverty rate, unemployment, ozone depletion, species extinction, greenhouse gas emission, collapse of fisheries, forest destruction, and many other factors have made sustainability a field of interest [3]. With the rapid growth in the world population, increase in prices of food and energy, and the subsequent recession, it is crucial to set up projects which will cater for human activities and for future generation. Consequently, the need of finding better measures on progress towards sustainability has become a major issue [4].

One of the ways to determine to which extent these adverse effects are occurring is to find the appropriate tool for measuring sustainability. Policy makers and experts are

Manuscript received August 16, 2013; revised September 27, 2013.

Pierre Olivier St Flour is with University of Technology, Mauritius (e-mail: ostrisaint@gmail.com). showing even more interests when it comes to addressing the new challenges raising from sustainability assessment at international, national and, regional level. To achieve their aims and objectives, they make use of readily available data from World Bank, World Health Organization, World Resources Institute and many other sources [5]. The data that are used within the various measurement tools are defined as sustainability indicators. So far many tools have been proposed to assess sustainability or any of its spheres but choosing the appropriate one is highly depended upon the research under study [6]. Some common approaches used by researchers in different fields of sustainability study include sustainability indicators analysis, multi-criteria decision making, system dynamics, fuzzy logic and, environmental or extended cost-benefit analysis, amongst others [7]. Due to the application of fuzzy logic concept in various fields of study, its capability to deal with imprecise information, mimicking human thinking and ability of deal with uncertainties of a system using set of parameters has made it a natural technical tool to measure sustainability [8]. Fuzzy logic presents an advantage both as compensatory and non-compensatory in one model at different context by using inferences through rules extracted from human experts. Compared to other existing models, it can make precise deductions without any mathematical description and is able to represent data in linguistic variables that is, in words or sentences, and is also capable of imitating human reasoning [9].

\section{Sustainability PERFORMANCE ASSESSMENT}

Sustainability performance assessment has become one of the main objectives of most environmental managers and decision-makers and now they are paying careful attention on how much information is available or missing to tackle the concept of sustainability. They are focusing on issues related to sustainability performance in order to understand how they are interrelated with lifestyles conditions and on finding ways on how to improve them [10]. When using incomplete or inaccurate information, facing the unsustainable path is much likely. It is important to understand human expectations and their contributions on the environmental, social, and economic issues such as pollution, energy consumption, land usage, food consumption, unstable economy, infant mortality rate, unemployment, criminal rate, public health expenditure, water usage and other factors which may give rise to a change in sustainability indicators. The assessment of the performance of a sustainability system necessitates a profound analysis of numerous parameters. The use of indicators is normally associated with other types of available information as the amount of information required about sustainable development is increasing considerably. 


\section{FUZZY LOGIC}

Fuzzy approach has been widely used in various fields of research and was found to be a powerful tool in different situations. Due to its capacity of mimicking human reasoning and predicting unforeseen results, it is one of the main reasons why it is extensively used by researchers [11]. It has also been found that it is able to handle very complex systems where mathematical representations are not possible [12]. The model presented here is describing how fuzzy approach has been applied to measure sustainability and is based on the three pillars of sustainability.

\section{SUSTAINABILITY INDICATORS}

Sustainability indicators should provide appropriate information for future objectives for social welfare, environmental quality and economic efficiency. For example, the way such indicators were used can determine answers for various situations as well as satisfying the objectives. Selecting the appropriate indicators can be a quite long development process. The procedures for sustainability assessment are highly dependent of indicators for better and accurate decisions. Using incomplete or inaccurate information of a particular indicator may affect the decision of any policy maker. An indicator normally allows seeing the state of a situation and the trend in which changes are occurred. Thus, using data collected from indicators, sustainability assessment can be generated under different conditions providing analytical deductions pertaining to the system under assessment. This is why fuzzy model was proposed to assess sustainable development index.

\section{Methodology}

The analytical method is divided into numerous sections. The aim is to assess sustainability. The first section shows the steps to normalize the raw data before fuzzification process takes place. The calculation is performed using the normalized data and finally the defuzzification process is carried out.

According to our methodology, the overall sustainability is composed of three modules: environmental (ENV sys), economic (ECON sys), and social sustainability (SOC sys).

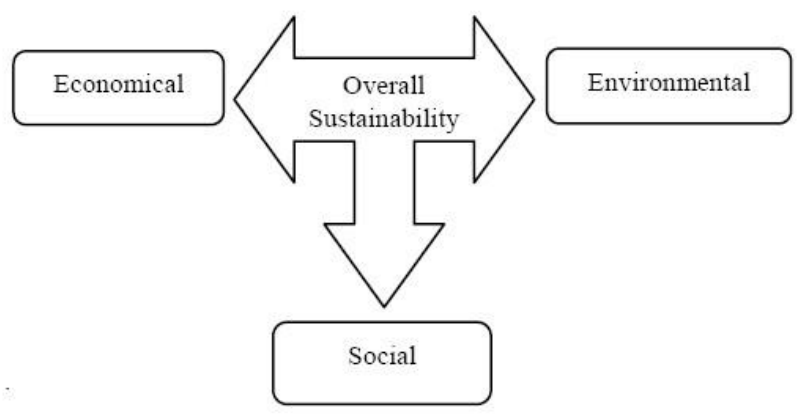

Fig. 1. Components of sustainability.

Fig. 1 shows the fuzzy evaluation method that was used to assess the sustainability of the three components: ENV sys, ECON sys, and SOC sys. Nine apropos sustainability indicators (I1 through I9) are gathered from the components and calculated. Finally, the results obtained are then interpreted and used to give out sustainability scenarios. The indicators can be grouped in inconsistent ways. The types of indicators are categorized as follows: pressure; status; and response [3].

1) Status describes the present state of a component chosen to be assessed such as life expectancy at birth.

2) Pressure is the changing force exerted on the status such as infant mortality rate.

3) Response summarises all actions taken to bring pressure to a level that will improve a better state, for example, public health expenditure.

In our model, the indicators of the sustainability components can be easily modified by replacing old ones to new ones according to new observations and series of data collected over a set period of time. Fig. 2 shows how the model has been configured.

\section{NORMALIZATION STEPS}

Allard et al. [13] stated that all available data should be normalized so as aggregate measure can be obtained to prevent anomalies while using raw data for each indicator directly. With this approach, data of each indicator are normalized to common membership grades between zero (least desirable value) and one (most desirable value). Basic indicators are represented by $i$ and $c_{i}$ is the value for a particular indicator that needs to be assessed. It has also a minimum value, $c_{\min }$, and a maximum value, $\mathrm{c}_{\max }$, respectively. The indicator has a target value denoted by $T\left(c_{i}\right)$ and it can be a single value or any interval range of values in the form of minimum and maximum $\left[T_{\min }, T_{\max }\right]$. Let $N\left(c_{i}\right)$ be the normalized value.

If $T_{i}$ corresponds to a maximum:

$$
N\left(c_{i}\right)=\left\{\begin{array}{l}
\frac{c_{i}-c_{\min }}{T\left(c_{i}\right)-c_{\min }} \\
1 \quad \text { for } c_{i} \geq T\left(c_{i}\right)
\end{array} \quad \text { for } c_{i} \leq T\left(c_{i}\right)\right.
$$

If $T_{i}$ corresponds to a minimum:

$$
N\left(c_{i}\right)=\left\{\begin{array}{l}
\frac{c_{\max }-c_{i}}{c_{\max }-T\left(c_{i}\right)} \\
1 \text { for } c_{i} \leq T\left(c_{i}\right)
\end{array} \quad \text { for } c_{i} \geq T\left(c_{i}\right)\right.
$$

If $T_{i}$ corresponds to an interval [ $\left.T_{\min }, T_{\max }\right]$ :

$$
N\left(c_{i}\right)= \begin{cases}\frac{c_{i}-c_{\min }}{T_{\min }-c_{\min }} & \text { for } c_{i} \leq T_{\min } \\ 1 \text { for } T_{\min } \leq c_{i} \leq T_{\max } & \\ \frac{c_{\max }-c_{i}}{c_{\max }-T_{\max }} & \text { for } c_{i} \geq T_{\max }\end{cases}
$$

\section{FUZZIFICATION STEP}

Each indicator should be computed to selected fuzzy sets with certain membership grades that define appropriate complete ordered partitions in $[0,1]$. All normalized sustainability indicators are defined using linguistic values. Each basic indicator is normalized and after fuzzification 
they are given linguistic values: " weak ," "medium" and " strong " as shown in Fig. 3 Sand " very bad ," " bad ," “ average ," good " and "very good" are assigned for composite indicators as shown in Fig. 4. These linguistic values are normally assigned to low normalize basic indicator values and are represented by fuzzy sets. The fuzzy set use membership functions. The fuzzy sets are assigned integer values $(0,1$ and 2) where 0 represent weak, 1 represent medium and 2 corresponds to strong.

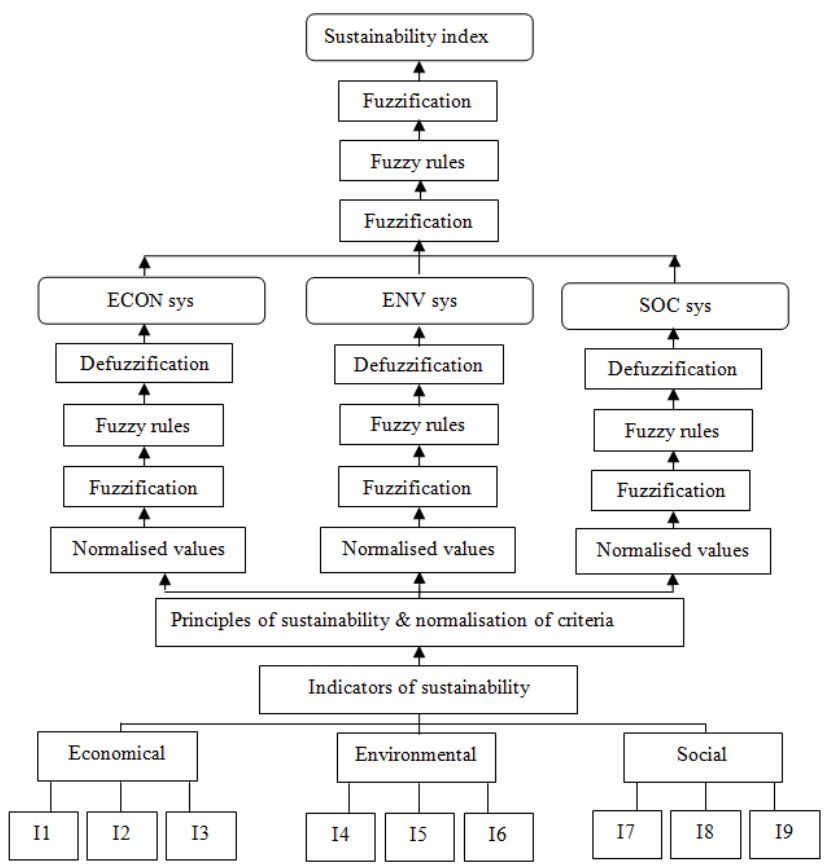

Fig. 2. Sustainability assessment framework.

Consequently, the same principles are applied to the five fuzzy sets. ( 0 corresponds to very bad, 1 corresponds to bad, and 2 correspond to average and so on).

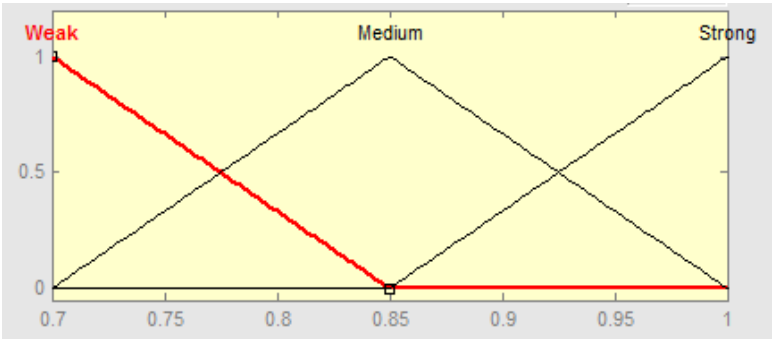

Fig. 3. Example of linguistic values of a basic indicator.

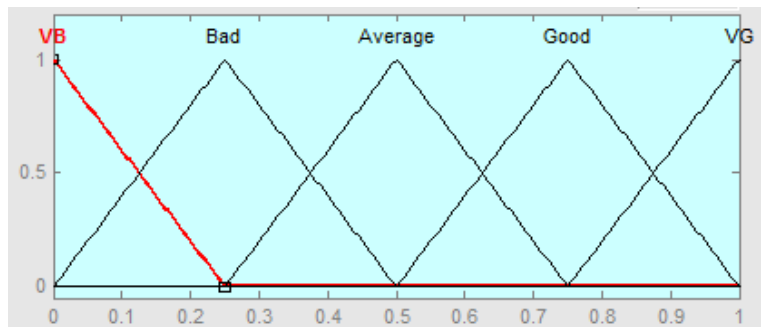

Fig. 4. Example of linguistic values and fuzzification of input variable.

\section{FUZZY RULE}

The fuzzy rules represent linguistically the dependence of composite indicators on other basic indicators. In order to determine the rules for sustainability, we have denoted possible solution of $3^{x}$ for the tertiary indicators where 3 represents the linguistic values discussed in the literature earlier for $x$ variables. For variable belonging to tertiary indicators, the rule base has $3^{2}=9$ rules because the maximum value for $x$ is 2 . The rules are listed below.

TABLE I: RULES FOR TERTIARY INDICATORS

\begin{tabular}{|c|c|}
\hline Rules & Basic indicators B1 \& B2 \\
\hline $\mathbf{R 1}$ & If $\mathrm{B} 1$ is weak and B2 is weak then RESULT is very bad \\
\hline $\mathbf{R 2}$ & If $\mathrm{B} 1$ is weak and $\mathrm{B} 2$ is medium then RESULT is bad \\
\hline $\mathbf{R 3}$ & If $\mathrm{B} 1$ is weak and $\mathrm{B} 2$ is strong then RESULT is average \\
\hline R4 & If $\mathrm{B} 1$ is medium and $\mathrm{B} 2$ is weak then RESULT is bad \\
\hline $\mathbf{R 5}$ & If $\mathrm{B} 1$ is medium and $\mathrm{B} 2$ is medium then RESULT is average \\
\hline R6 & If $\mathrm{B} 1$ is medium and $\mathrm{B} 2$ is strong then RESULT is good \\
\hline $\mathbf{R 7}$ & If $\mathrm{B} 1$ is strong and $\mathrm{B} 2$ is weak then RESULT is average \\
\hline $\mathbf{R 8}$ & If B1 is strong and B2 is medium then RESULT is good \\
\hline R9 & If B1 is strong and B2 is strong then RESULT is very good \\
\hline
\end{tabular}

\section{DEFUZZIFICATION PROCESS}

Defuzzification is the final operation where fuzzy values can be corrected to single crisp values. As defuzzification method, the center-of-gravity formula will be used as follows:

$$
X_{\text {crisp }}=\frac{\sum_{i}=\text { lin.var. }(P V)_{i} i}{\sum_{i}=\text { lin.var. } \mu_{\mathrm{i}}}
$$

where $(P V)_{i}=$ peak value of linguistic variable $i$

After fuzzy reasoning, crisp value will be derived from linguistic output variable that was obtained. Finally, for representing fuzzy value obtained from the above mentioned output variables, single crisp numeric value should be derived.
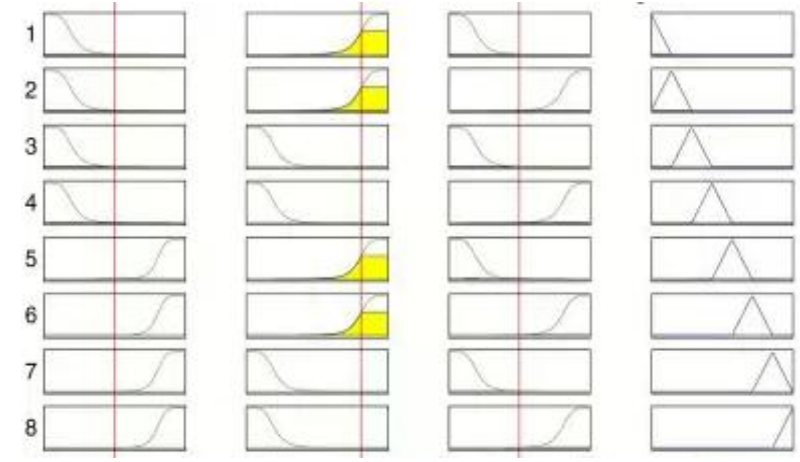

Fig. 5. The calculus for overall sustainability.

The selection of indicators in the normalized form is fuzzyfied. Afterwards, the final results obtained from the model represent the degree of sustainability of a given system under assessment, for example, a country, using corresponding set of variables. The three fuzzy variables are 
then subjected to inference rules that the secondary variables are computed. Fig. 5 above shows the computation of the overall sustainability and the generated output.

The proposed model is very flexible and policy makers are able to choose their appropriate indicators according to their needs and it allows them define the set of rules for the system they want to assess. The model can be modified and new inputs can be added as reality changes depending on statistical indicators after including the experience change.

\section{Discussions}

The impact of sustainability on environmental, economic, and social systems emphasize the urgent need to find a standard framework for investigating and measuring sustainable development. Such framework should encompass the environmental, economic and societal sustainability. Many assessment tools have been used to measure sustainability but managing it effectively becomes even more difficult when the scope of measurement reach national or international level. Consequently, policy makers need a methodological approach to deal with such issues. Fuzzy logic is best suited in these circumstances. Due to its capability of dealing with uncertainties, imitate human reasoning, and provide expected output which is expressed in linguistic terms, it is qualified as an intelligent tool that can be used for sustainability assessment. The model aimed at describing the different steps in applying fuzzy logic as an analytical tool for assessing sustainability. The model can be adapted to any type of conditions according to sets of parameters used for a particular system. These parameters can be removed and replaced by new ones to a given set of conditions, for example, factors causing detrimental effects in the climatic condition. Many proposed systems and tools used to assess sustainability lack full knowledge of the system under assessment. Sustainability seemed vague to many experts and decision makers since they did not have complete knowledge of how could human factors affect the ecological system. Fuzzy logic approach compensates a complete understanding of a system.

The proposed fuzzy approach is able to predict future sustainability of a given system. The model also helps to overcome situations with difficulties arising when assessing certain criteria. With the application of fuzzy logic, many areas can be assessed and derived information can be compared over time as it is easy to use and interpret. Hence, sustainability can be viewed in a much optimistic way. Finding appropriate indicator is another key factor of fuzzy models. Data collected for basic indicators is available for many countries from various resources but policy makers should consider certain priorities towards sustainable decision making. For example, if a basic indicator is found to be positive, an increase in its values will promote sustainability.

A potential problem in applying fuzzy model effectively is the nature in which fuzzy rules have been formulated. For example, the assessment of a sustainable indicator, $\mathrm{x}$, using two linguistic values will result with a number of fuzzy rules base $2^{x}$ rules. On the other hand, an increase in the number of sustainable indicators will lead to an exponential increase in the number of fuzzy rules as well. Since there is an exponential increase, the fuzzy rules become difficult to use if not properly defined. A possible remedial action is to choose amongst promoting indicators and ultimately use them independently. In other words, if there is a strong relationship between two indicators, then eliminating both of them and replacing them by a new one will fit the situation. This is why fuzzy logic is found to be a practical tool in sustainability measurement.

\section{CONClusion AND PeRsPectives}

The model represents a description of the concept of sustainability. Most existing methods focused purely on environmental or social approaches. These methods are widely accepted but they should incorporate all sustainability concepts. The model studied and proposed comparatively composes of economic, environmental and social concepts and provides a clear picture of sustainable development. Policy makers can use this model as a practical tool at international, national and regional level. To apply this model effectively, it will necessitate a good definition of indicators under assessment, and good definitions the fuzzy rule bases as well. It is also important to know that such a model permit modification of indicators under some changing conditions. Policy makers can adjust input data to analyze the effects and predict a sustainable future.

\section{ACKNOWLEDGMENT}

The authors would like to thank all the people for the outstanding support for this research.

\section{REFERENCES}

[1] R. Lozano, "Envisioning Sustainability Three dimensionally," The Journal of Cleaner Production, vol. 16, pp. 1838-1846, 2008.

[2] I. Erol, S. Sencer, and R. Sari, "A new fuzzy multi-criteria framework for measuring sustainability performance of a supply chain," The Journal of Ecological Economics, vol. 70, no. 6, pp. 1088-1100, 2011

[3] Y. A. Phillis and L. Andriantiatsaholiniaina, "An ill-defined concept and its assessment using fuzzy logic," The Journal of Ecological Economics, vol. 37, no. 3, pp. 435-456, 2001.

[4] A. L. Dahl, "Achievements and gaps in indicators for sustainability," The journal of Ecological Indicators, vol. 17, pp. 14-19, 2012.

[5] D. Hîncu, "Modeling the urban sustainable development by using fuzzy sets," Theoretical and Empirical Researches in Urban Management, vol. 6, no. 2, pp. 88-103, 2011.

[6] M. Pislaru and A. Trandabat, "Assessment of Company Environmental Impact using Fuzzy Logic," in Proc. The International Conference on Environment Science and Engineering (IPCBEE '12), 2012, vol. 32, pp. 97-102.

[7] S. Muller, "Evaluating the sustainability of agriculture: the case of the Reventado River Watershed in Costa Rica," European University Studies, Series 5, Economics and Management Peter Lang, Germany, vol. 2194, pp. 223, 1997

[8] T. M. Kirankumar and M. A. Jayaram, "Natural Computing in Spatia Information Systems," in Proc. $2^{\text {nd }}$ National Conference on Challenges \& Opportunities in Information Technology (COIT-2008, 2008), pp. 262.

[9] M. Pislaru, A. Trandabat, and S. Avasilcai, "Environmental Assessment for Sustainability Determination based on Fuzzy Logic Model," in Proc. $2^{\text {nd }}$ International Conference on Environmental Science and Technology (IPCBEE '11), 2011, vol. 6, pp. 301-305.

[10] W. Jin, L. Xu, and Y. Yang, "Modeling a policy making framework for urban sustainability: Incorporating system dynamics into the Ecological Footprint," The Journal of Ecological Economics, vol. 68, no. 12 , pp. 2938-2949, 2009. 
[11] F. Gagliardi, M. Roscia, and L. Lazaroiu, "Evaluation of sustainability of a city through fuzzy logic," vol. 32, pp. 795-802, 2007.

[12] M. Pislaru, A. Trandabat, and S. Avasilcai, "Environmental Assessment for Sustainability Determination based on Fuzzy Logic Model," in Proc. $2^{\text {nd }}$ International Conference on Environmental Science and Technology (IPCBEE '11) , 2011, vol. 6, pp. 301-305.

[13] F. Allard, F. Chéqui, E. Wurtz, and L. Mora, "A Methodology to Assess the Sustainability of Rehabilitations Projects in Urban Buildings," LEPTAB, University of La Rochelle: La Rochelle, France, 2004.

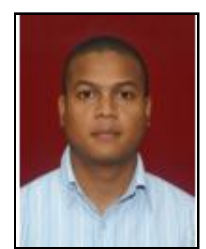

Pierre Olivier St Flour was born on Mauritius on November 11, 1984. He graduated with a BSc. (Hons) in Information Technology Enabled Services in 2008 and a MSc. in Business Enterprise Systems in 2011 from the University of Technology, Mauritius. He is now pursuing an MPhil/PhD in Sustainable Development and his main research interest is developing a measurement tool for assessing sustainable development at country level basically in Mauritius. 